\title{
IRREGULAR PERIOD VARIATIONS OF ECLIPSING BINARIES
}

\author{
TIBOR J. HERCZEG \\ Department of Physics and Astronomy \\ University of Oklahoma \\ Norman, Oklahoma 73019, USA
}

\begin{abstract}
Considering a small number of representative systems among them $U$ Cephei, SV Centauri, V471 Tauri, EM Cygni, the case is made for irregular period variations playing a substantial role in the observed changes of close binary periods.
\end{abstract}

Eclipsing systems with regular, well-understood, geometric type of variations of the period (such as apsidal rotation or light-time effect) represent only a handful of objects, probably no more than 30-35 systems. For most of the other close, interacting systems with intrinsic variations of the period, theory suggests period changes which for the limited time of observations would appear as approximately linear variations with time, increases or decreases corresponding to a constant rate of mass flow between the components. Individual observers and also surveys and statistical studies frequently find these types of linear variations which manifest themselves in quadratic time variations of the observed residuals.

This short paper intends to warn against rushing to general statements concerning linear period variations. The number of well-established cases are far fewer than one would expect; irregular variations, frequently in the form of discontinuous changes of the period or phase, play a significant role. In a number of cases these variations may be explicable by irregularities of the light curve but characteristically, even if progressive, secular variations are clearly established, they are in most cases intertwined with sudden and irregular changes.

Limitations of space make it impossible even to attempt a demonstration of this cautionary statement. Half a dozen examples may serve as mere illustration. It is perhaps interesting to remind the reader that the only case where a linear period variation (decrease) and its interpretation (gravitational radiation) may be accepted without reservation is, in my opinion, the radio binary pulsar PSR $1913+16$, see Taylor and Weisberg (1982). Clearly, photometric and spectroscopic determinations can still not compete with the superior accuracy of pulsar timing but we should mention at least one famous eclipsing binary observed over the last 200 years. The system $\beta$ Lyrae shows a monotonous increase in the period the rate of which was object of much conflicting descriptions. It was therefore a pleasant surprise when Klimek and Kreiner (1973) succeeded in describing the period variations using a simple quadratic ephemeris formula, i.e. introducing a "steady" linear increase of the period at the rate of $18 \mathrm{~s} / \mathrm{yr}$. It should be mentioned, however, that an alternative third order formula gives almost the same overall accuracy. 
1. To illustrate these possibilities we gave in Fig. 1ab the miniaturized juxtaposition of the O-C diagrams of two rather ordinary, undistinguished W UMa systems, V401 Cygni and $Y$ Sextantis; the diagrams are taken from a more detailed discussion which goes to the printer in these days. V401 Cyg stands for the few short period contact systems -certainly the minority of cases -- which show an acceptable quadratic representation of the residuals, that is, an approximately linear variation (increase) of the period. This trend is still holding as a recent timing of primary minimum (July 2, 1991) seems to indicate, kindly observed at our request at the National Undergraduate Research Observatory near Flagstaff, Arizona.
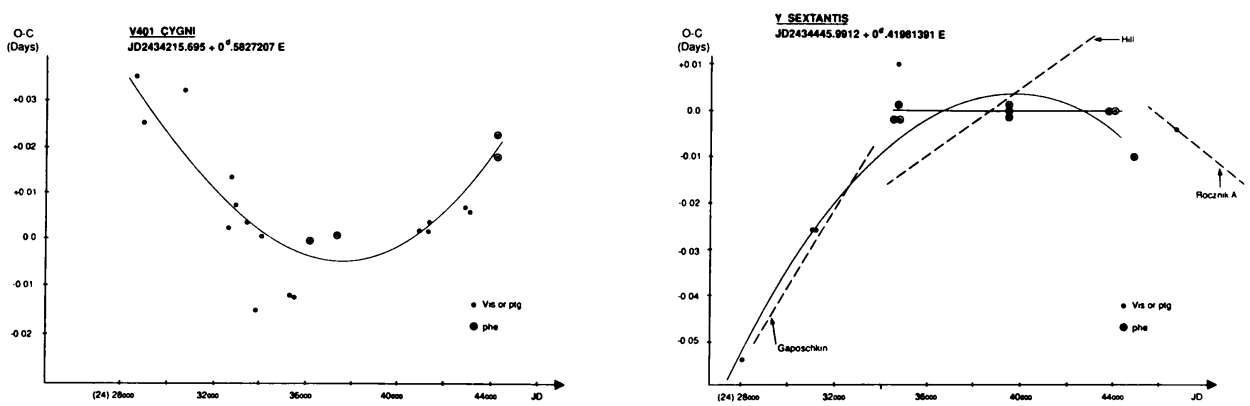

Figure 1ab. O-C diagrams for V401 Cyg and Y Sex, from an unpublished manuscript of the author.

The system Y Sex is a very different case although the O-C diagram, at first glance, may suggest a linearly decreasing period. A closer look, however, clearly shows the lack of a simple smooth representation: deviations amounting to $0.004-0.007$ days from a parabolic fit are not acceptable for photoelectric timings. The case can be made for an interval of constant period between the observations made at Tokyo Observatory (1953/54) and those at Kitt Peak (1979), yet unpublished. With this interpretation the period shows irregular variations and, in particular, two possibly abrupt changes.

This description of the period variations is by no means far-fetched or merely an ad hoc hypothesis. All types of eclipsing variables, especially algols and contact systems can show such variations: relatively sudden period changes, of the order of $10^{-5} \mathrm{P}$, with long intervals of practically constant period between these "jumps." Sections of constant period may last from a few years to as long as 20-25-30 years. A good example is Algol itself and the reader is requested to look up Fig. 5 in the discussion by Frieboes-Conde et al. (1970). It is as the mass flow had been reduced for several years to a trickle and then revived for a short while, for months, perhaps weeks. This type of period variation plays a prominent role in W UMa systems -- in the recent literature, major period changes of this nature have been reported in the case of TZ Boo, SW Lac, AH Vir, among others. It is also very instructive to see in Hershey's seminal paper (1975) about the astrometry of VW Cep, how the smooth O-C curve turns, after subtraction of the light time effect, into an irregular zigzag line, see Fig. 2 of the paper.

We have to add that in some, perhaps in many, cases O-C curves may show spurious variations due to strong distortions of the light curve; this is so, in particular, for the RS CVn-stars with their notoriously disturbed light curves. Nevertheless, I am convinced that real period variations do occur, certainly in the majority of cases studied. 
2. Even if for a system the existence of a secular, progressive variation of the period can be demonstrated, the most accurate observations show, more often than not, the secular variations interspersed with irregular, mostly discontinuous period changes. A case in point is the much studied "active algol" $U$ Cephei. The basic catalogue data, period, spectral types etc. are not too dissimilar to those of Algol itself but the two systems are obviously in quite different phases of binary evolution.
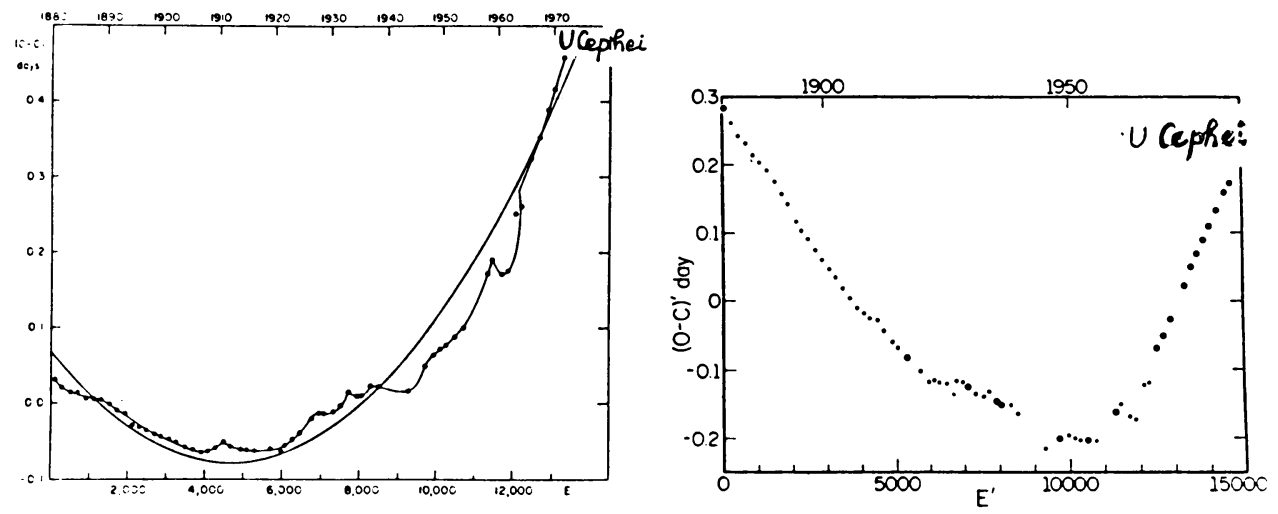

Figure 2ab. U Cephei O-C diagrams. a by D. S. Hall, b by A. H. Batten.

A review of the photometric behaviour of $U$ Cep is given by Olson (1985); as to the spectroscopy, we may rely on Batten's earlier paper (1974). The secular increase of the period is unquestionable, as the rather unusual O-C curve in Fig. 2a illustrates. The unusual small scale variations, the "fine structure" of the O-C diagram are, however, almost completely ironed out by rigorous averaging in Fig. 2b. After 1970, if we consider the normal points of Fig. $2 b$ only, a virtually constant period emerged at 2 d493060. Yet as our Fig. 3, taken from a study of Olson et al. (1981), clearly shows, irregular variations persisted even during that time. The "event" (sudden decrease of the period) in 1974 seems to have been accompanied by enhanced "activity" in the system. On the other hand, such events may cause distortions of the light curve, which again may lead to spurious timings of the eclipses and also spurious variations of the period. The problem was paid much attention, see Crawford and Olson (1979), Olson et al. (1981), with the result that photometric disturbances near minima tend to delay the apparent phase of primary eclipse; plausible models have been worked out to explain these findings. Nonetheless, the undisturbed eclipses show convincingly the reality of the irregular period changes. One has to admit, though, that the number of distorted minima is almost embarrassingly large.

A somewhat similar case is SV Centauri, an early type system of 1.66 day period and $\beta$ Lyrae-type light curve. The change of the period is among the fastest observed: a monotonous decrease at an average rate of $-3.1 \mathrm{sec} /$ year, corresponding to $\mathrm{P} / \mathrm{P} \approx 46000$ years! The rate of decrease, however, is far from constant. An early study found such a regularity in alternating changes of $\mathrm{dP} / \mathrm{dt}$ that a sine-curve was proposed for its representation -- due to light time effect -- superposed on a linear decrease of the period (O'Connell 1951). A recent discussion by Herczeg and Drechsel (1985) indicates, 


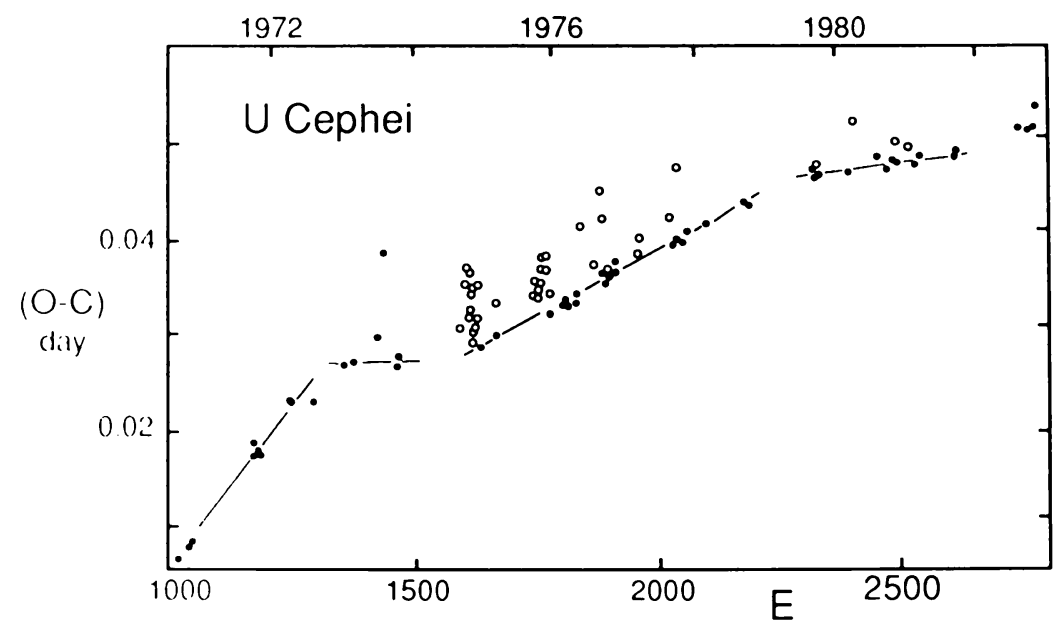

Figure 3. U Cephei, a section of the O-C diagram. Dots: undisturbed eclipses. Circles: disturbed eclipses.

however, that variations of $\mathrm{dP} / \mathrm{dt}$ are less regular and seem to show abrupt transitions; there is a tendency to alternating changes. The reader is urged to take a look at Fig. 7 on p. 12 of the paper cited.

Like U Cep, SV Cen shows secular variations quite clearly, but a "fine structure" is manifestly present in both $\mathrm{O}-\mathrm{C}$ diagrams and its closer study may well contribute to our understanding of the actual mechanism of the mass exchange.

3. The famous "white dwarf binary" V471 Tauri, discovered by Nelson and Young in

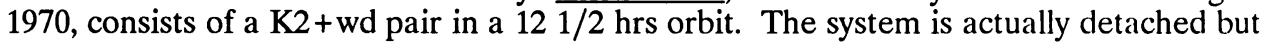
it is widely considered a possible progenitor of a cataclysmic binary, a much closer pair. In the literature it is occasionally stated that the period is, indeed, decreasing. Such a statement, however, is not compatible with available evidence as a glance at Fig. 4 clearly shows.

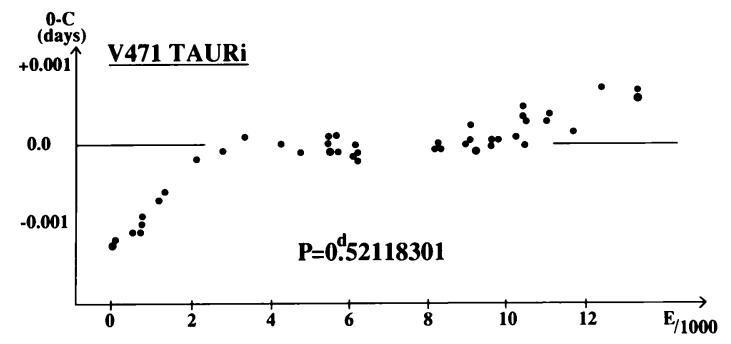

Figure 4. O-C values for V471 Tau, see text. Some of the points represent two nearly identical residuals.

Here we plotted the residuals of 48 representative timings of the minimum against the ephemeris

$$
\text { Min.I = JD2442362.8046 + 0d52118301.(E-3663); }
$$

the minimum epochs have been selected from the lists given by Young et al. (1983), 
Ibanoglu and Evren (1984), Skillman and Patterson (1988) and Ibanoglu (1989). There is a long interval, more than 10 years, of a virtually constant period: for this stretch of time, the average $\mathrm{O}-\mathrm{C}$ value is $-0 \mathrm{~d} 00003$, with a standard deviation of $0 \mathrm{~d} 000083$ or $7.2 \mathrm{sec}$. Before and after this interval of constant period, the period was different but the difference between the values indicated for 1970 and 1989 is merely of the order of -0.016 sec, hardly enough to support a claim of decreasing period. The first transition might have been abrupt (see also Ibanoglu and Evren, their Fig. 2).

On the other hand, the nature of the O-C curve, the apparent inflection around $E=7000$, remind us of a possible light time effect. Yet as Ibanoglu points out in his 1989 paper, recent minima tend to deviate from the latest prediction based on the light time hypothesis (Beavers et al. 1986). Thus it is safer to say that the period of V471 Tau is at present not well understood; it certainly shows no tendency to decrease but it may show irregular variations.

The light curve of V471 Tau is known to be very strongly disturbed and variable. Nevertheless, the timing of the relatively deep well-like eclipses due to the occultation of the white dwarf compoennt, the persistence of a period over years, the independence of the time residuals from small variations of the eclipse duration (cf. the careful study of Stillman and Patterson), all speak for the reliability of the O-C curve presented here.

4. Concerning the cataclysmic variables themselves, (this subclass of the eruptive variables comprising novae, recurrent novae, dwarf novae and nova-like variables) the main question is: do the observed period changes contribute to the theory of their origin and evolution? The answer is: very little, if at all. Our best support is the distribution of the periods, not their variations.

The cataclysmics are difficult to observe, requiring more sophisticated techniques than most other eclipsing binaries. Furthermore, the light curves are unruly and variable and the phase stability of minima may be lessened due to the circumstance that the deepest portion of the eclipse frequently corresponds to the occultation of a "hot spot," not the white dwarf component. The residuals scatter considerably, $0.001,0.002$ days and most of the resulting $\mathrm{O}-\mathrm{C}$ diagrams remain ambiguous.

An earlier review by J. Pringle (1976) of nine such systems is still characterizing the observational situation. In seven of the nine cases Pringle found the existence of linearly decreasing or increasing periods "not clear cut as previously suggested." Only two systems, N. Aurigae 1891 (T Aur) and EM Cygni seem to exhibit significant period changes (negative $\mathrm{dP} / \mathrm{dt}$ in both cases). Even here, the description of the period of $\mathrm{EM}$ Cygni may require a reconsideration, as our Fig. 5 suggests. Pringle's parabolic representation is of very high significance, at 99.8 per cent. The scatter of data is considerable and the author added the cautionary remark: "The reader may judge the credibility of a 99.8 per cent result." A few new observations, indeed, do not conform with the proposed formula. Although a quadratic formula will, in general, lead to smaller residuals than a linear one, it is perhaps safer to conclude that for EM Cyg no definite change of the period has been found yet.

A certain danger lies in the circumstance that anyone who only looks at the number, the 99.8 per cent, will interpret this level of significance as virtual certainty. How many misleading or premature data referring to period variations and mass transfer rates might have crept in the various catalogues and surveys? 


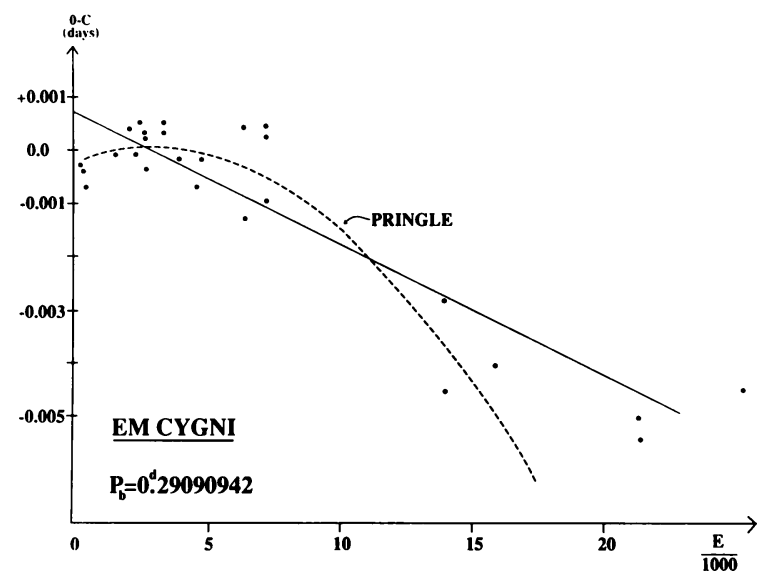

Figure 5. Residuals for the dwarf nova EM Cyg; the last four points represent observations since 1975.

Conclusions. Irregular, oft discontinuous variations play a substantial role in period changes of close binaries; this role seems to be enhanced in contact or near contact systems. Even if a secular trend of period variations is unmistakably present, frequently we find a "fine structure" that needs explanation. For the important class of cataclysmic binaries, no clear cut evidence for preferential variations of the period seems to be traceable, as yet.

In general, the so-called open and shut cases are far less numerous than references in the astronomical literature would make one expect. After about 40-45 years of intense and reasonably accurate studies, comprehensive understanding of intrinsic period variations in close binary systems may still elude us.

Acknowledgement. My thanks to Jaquine Hudson Milivojevic for preparing this manuscript. 


\section{REFERENCES}

Batten, A. H. 1974, Publ. Dom. Astrophys. Obs. 14, 191

Beavers, W. I., Herczeg, T. J., Lui, A. 1986, Astrophys. J. 300, 785

Crawford, R. C. and Olson, E. C. 1979, Publ. Astr. Soc. Pacific 91, 111

Frieboes-Conde, H., Herczeg, T. J., Hoeg, E. 1970, Astron. Astrophys 4, 78

Herczeg, T. J. and Drechsel, H. 1985, Astrophys. Space Sc. 114, 1

Hershey, J. L. 1975, Astron. J. 80, 662

Ibanoglu, C. and Evren, S. 1984, Inf. Bull. Var. Stars No. 2573

Ibanoglu, C. 1989, Astrophys. Space Sci. 161, 221

Klimck, Z. and Kreiner, J. M. 1973, Acta Astr. 23, 231

O'Connell, D. S. J. 1951, Riverview Coll. Obs. Publ. 2, 69

Olson, E. C. 1985, in "Interacting Binaries" (eds. P. P. Eggleton and J. E. Pringle), Reidel Publ. Co.

Olson, E. C. et al., 1981, Publ. Astr. Soc. Pacific 93, 464

Pringle, J. E. 1975, Monthly Not. RAS 170, 633

Skillman, D. R. and Patterson, J. 1988, Astron. J. 96, 976

Taylor, J. H. and Weisberg, J. M. 1982, Astrophys. J. 253, 308

Young, A. et al. 1983, Astrophys. J. 267, 655 